\title{
PESQUISA DE CONSUMO DE BATATAS PROCESSADAS E DESENVOLVIMENTO E ANÁLISE SENSORIAL DE BATATA FUNCIONAL PRÉ-ASSADA
}

\author{
CONSUMER RESEARCH OF PROCESSED POTATOES AND \\ DEVELOPMENT AND SENSORY ANALYSIS OF PRE-ROASTED \\ FUNCTIONAL POTATO
}

\begin{abstract}
Alaides Sanae Suguiura ${ }^{1}$, Louise Marçal Matuszevski ${ }^{1}$, Frederico Rodrigues Lovato $^{2}$, Sabrine Zambiazzi da Silva ${ }^{3}$, Daniela Miotto Bernardi ${ }^{*}$

${ }^{1}$ Nutricionista, Centro Universitário Fundação Assis Gurgacz (FAG). ${ }^{2}$ Engenheiro Químico, FUNDETEC. ${ }^{3}$ Nutricionista. Mestre, Docente do curso de Nutrição, Centro Universitário da Fundação Assis Gurgacz (FAG). ${ }^{4}$ Nutricionista Doutora, Docente do Curso de Nutrição, Centro Universitário Fundação Assis Gurgacz (FAG)
\end{abstract}

* Autor correspondente: danimiottober@gmail.com , https://orcid.org/0000-0001-9019-3835

\section{RESUMO}

O presente estudo teve como objetivo estudo foi aplicar a pesquisa de consumo de batatas processadas e alimentos funcionais, bem como desenvolver e realizar a caracterização físico-química, microbiológica e sensorial de produtos funcionais similares à batata smile. A pesquisa de consumo foi realizada via Google Drive, sendo composta por $1^{\circ}$ questões fechadas. Foram elaboradas 3 formulações diferentes, uma com adição de $20 \%$ linhaça (BFL), a segunda com $20 \%$ de aveia (BFA) e a terceira com $10 \%$ de linhaça e $10 \%$ de aveia (BFLA). Foram realizadas análises microbiológicas, físico-químicas e sensorial. A pesquisa de consumo e a análise sensorial foram realizadas com 257 e 134 indivíduos, respectivamente. Entre os produtos de batatas processadas e derivados, a batata frita foi apontada como mais consumida (46,7\%). Entre os provadores, $69,3 \%$ acham a batata um alimento saudável e $74,3 \%$ relatam não achar a batata smile saudável. Em relação ao produto desenvolvido, as análises microbiológicas se mantiveram dentro do exigido pela legislação, bem como foi possível observar redução de carboidrato, aumento de lipídios, fibras e proteína. Os resultados da análise sensorial mostraram que houve diferença significativa entre as amostras, a $B C$ foi a formulação mais aceita, obtendo um IA (\%) de $90 \%$, comparado a $69 \%$ BFA, 64\% BFAL e $60 \%$ BFL. Portanto, o desenvolvimento de produtos inovadores e saudáveis neste setor tem grande relevância devido ao consumo acentuado de batatas processadas.

Palavras-chaves: Batata smile, aveia, linhaça e alimentos funcionais

\begin{abstract}
The objective of the present study was to study the consumption of processed potatoes and functional foods, as well as to develop and perform the physical-chemical, microbiological and sensorial characterization of functional products similar to potato smile. The consumer research was conducted via Google Drive, and it was composed of ten closed questions. Three different formulations were produced, one with $20 \%$ linseed (FPL), the second with $20 \%$ oat (FPO) and the third with $10 \%$ linseed and $10 \%$ oat (FPLO). Microbiological, physical-chemical and sensorial analyzes were performed. Consumer research and sensory analysis were performed with 257 and
\end{abstract}


134 subjects, respectively. Among the processed products and potatoes derived, potato chips were rated as the most consumed (46.7\%). Among the tasters, $69.3 \%$ find the potato a healthy food and $74.3 \%$ report not finding the potato smile healthy. In relation to the product developed, the microbiological analyzes remained within the required by the legislation, as well as it was possible to observe reduction of carbohydrate, increase of lipids, fibers and protein. The results of the sensorial analysis showed that there was a significant difference between the samples, the BC was the most accepted formulation, obtaining an acceptability index (\%) of $90 \%$, compared to $69 \%$ FPO, 64\% FPLO and 60\% FPL. Therefore, the development of innovative and healthy products in this sector has great relevance due to the accentuated consumption of processed potatoes.

\section{INTRODUÇÃO}

O conceito de alimentos funcionais originou-se por volta dos anos 80 no Japão, pelo fato de conter ingredientes que trazem benefícios às funções do organismo (SCHULKA e COLLA, 2014). No Brasil, segundo a Agência Nacional de Vigilância Sanitária (1999), o alimento ou ingrediente com propriedades funcionais são aqueles que além de oferecer funções nutricionais básicas, produzem efeitos fisiológicos e/ou metabólicos e/ou efeitos benéficos à saúde, assegurando o consumo seguro sem supervisão médica.

Conforme o autor Silva et al, (2016), os alimentos funcionais são considerados uma nova tendência sobre os comércios alimentícios, devido à procura da população por dietas saudáveis, sendo representados como um nicho de mercado altamente lucrativo, pelo fato do alto valor agregado e com marketing intenso na busca pelo consumo. Em contrapartida, Food Trends Brasil (2010), apresenta, segundo uma pesquisa realizada com a população, apenas $8 \%$ dos indivíduos relatam conhecer bem o termo alimentos funcionais e $40 \%$ diz nunca terem ouvido falar sobre o termo.

As fibras alimentares são consideradas alimentos funcionais, uma vez que, podem contribuir para a diminuição do risco de doenças crônicas não transmissíveis, como diabetes, doenças cardiovasculares e doenças relacionadas ao trato gastrointestinal (COSTA; ROSA, 2016). As fibras alimentares são classificadas de acordo com a sua viscosidade, fermentação microbiana no intestino grosso e a sua solubilidade em água, podendo ser classificada em fibras solúveis ou insolúveis (COSTA; ROSA, 2016).

A aveia considerada uma gramínea anual, pertence à família Poaceae, tribo Aveneae, com o gênero Avena. As principais espécies presentes no país são a aveia sativa, que teve sua origem na Ásia, a aveia Byzantina, e a aveia Strigosa, originandose na Ásia antiga (EMBRAPA, 2012; SIMONATTO, 2013). A mesma é considerada uma fibra solúvel, além de ajudar na diminuição do colesterol, apresenta um possível efeito protetor para a redução de risco de câncer intestinal e na redução da absorção da glicose em indivíduos com diabetes (BERNAUDI; RODRIGUES, 2013).

A linhaça é uma semente resultante do linho (Linum usitatissimum L.), pertencente à família das lináceas, originou-se na Ásia (STANCK.T. L, 2015). A própria, apresenta elevado teor de Ácidos graxos poli-insaturados (AGPL), principalmente o ácido a-linolênico (ALA), fibras solúveis, proteína vegetal, flavonoides, lignanas e ácidos fenólico. O conteúdo de ALA presente se destaca pelo fato de evitar obstrução nas artérias, diminuindo o risco de problemas cardíacos, além da redução do estresse oxidativo (PILAR, 2014). 
Os produtos processados são considerados uma alternativa prática e favorável, apesar do seu custo e sabor menos palatável comparado a uma refeição caseira (AGROCLUSTER, 2015). De acordo com os estudos realizados sobre o perfil de consumo no Brasil, o termo conveniência e praticidade com $34 \%$ é o principal fator decisivo para o consumo alimentar, seguido de confiabilidade / qualidade e sensorialidade / prazer com $23 \%$, por último, a saudabilidade, bem-estar, sustentabilidade e ética com 21\% (AGROCLUSTER, 2015).

Entre os produtos processados, as batatas congeladas destacam-se em relação à preferência de consumo pela população. Esse aumento no consumo se deu a cerca de 20 anos, quando se iniciou a necessidade de maior agilidade nos restaurantes, desta forma, para atender a demanda do mercado, as empresas estão desenvolvendo produtos com incorporação de ingredientes funcionais em produtos já existentes (RAMOS, SILVA E DUMBRA, 2013.; NOBRE, 2013).

Com tudo, define-se batatas congeladas como produtos obtidos a partir do processamento de lavagem, descascamento e corte, submetidas a pré-frituras, armazenadas sob condição de congelamento a $-18{ }^{\circ} \mathrm{C}$ (NETO, 2016). Há estimativas de 164 mil toneladas de batatas in natura que são utilizadas para a produção de batatas congeladas (EMBRAPA, 2015). Apesar das batatas in natura serem utilizadas para a produção de batatas congeladas, as batatas processadas são consideradas um alimento ultra processado, apresentando uma grande quantidade de densidade energética, açúcar, sódio, gorduras trans ou saturadas e deficiente de fibras dietéticas, contribuindo para o ganho de peso, aumento do risco de doenças crônicas não transmissíveis (NEVES, 2016).

O objetivo do presente estudo foi aplicar a pesquisa de consumo de batatas processadas e alimentos funcionais, bem como desenvolver e realizar a caracterização físico-química, microbiológica e sensorial de produtos funcionais similares à batata smile.

\section{METODOLOGIA}

\subsection{CRITÉRIOS ÉTICOS E POPULAÇÃO}

A pesquisa foi realizada de acordo com os procedimentos exigidos para a coleta de dados, respeitando os critérios éticos, o projeto foi encaminhado ao comitê de ética e pesquisa com seres humanos do Centro Universitário Assis Gurgacz (FAG), recebendo parecer favorável de número 1.879.790. Todos os indivíduos antes de participarem da pesquisa foram convidados a ler e assinar o termo de consentimento livre e esclarecido, é importante ressaltar que todos os participantes apresentavam igual ou acima de 18 anos.

\subsection{PESQUISA DE CONSUMO}

O questionário aplicado via Google Drive foi composto por 10 perguntas fechadas, o mesmo foi entregue aos participantes a partir do link, divulgado via redes sociais (Facebook e WhatsApp). Assim, os participantes entravam no link enviado, realizavam a leitura e posteriormente respondiam as questões sem qualquer constrangimento ou coação.

Foram questionados sobre o consumo de batatas e batatas processadas, frequência de consumo, o motivo de consumo, percepção em relação a saudabilidade da batata e de batata smile, conhecimento sobre os benefícios das fibras e o seu consumo, hábitos de consumir alimentos enriquecidos e benefícios dos alimentos funcionais. 


\subsection{ELABORAÇÃO DE PRODUTOS FUNCIONAIS SIMILARES À BATATA SMILE}

Foi utilizada como matéria-prima batatas inglesas, aveia, linhaça, amido de milho, sal e leite adquiridos no mercado local também utilizou-se essência de batata (referência: DAR1359, ingrediente: destrina, sal refinado, realçador de sabor glutamato monossôdico, queijo em pó, antiumectante dióxido de silício e aromatizantes idênticos aos naturais), doada pela empresa Doremus. Os ingredientes utilizados e suas proporções estão indicados na Tabela 1.

Os ingredientes foram pesados em balança analítica para o preparo de cada formulação. A batata foi previamente cozida, em panela de pressão comum e amassada, os ingredientes foram incorporados à massa até homogeneização da mesma e após, moldadas para obtenção da forma desejada (smile). A Figura 1 mostra as etapas de preparo do produto.

Figura 1. Fluxograma do processamento de produtos funcionais similares à batata smile

Batatas cozidas e demais ingredientes
$\downarrow$
Homogeneização
$\downarrow$
Moldagem
$\downarrow$
Pré-assar
$\downarrow$
Congelar

Tabela 1. Formulação das amostras de produtos funcionais similares à batata smile com variação na concentração de aveia e linhaça, sendo BFL (somente farinha de linhaça e linhaça dourada), BFA (somente farinha de aveia e aveia em flocos) e BFLA ( $50 \%$ aveia e $50 \%$ linhaça).

\begin{tabular}{cccc}
\hline Ingredientes & BFL \% & BFA \% & BFLA \% \\
\hline Batata & 67 & 67 & 67 \\
Amido de milho & 1,4 & 1,4 & 1,4 \\
Farinha de aveia & - & 17 & 8,5 \\
Aveia em flocos & - & 3 & 1,5 \\
Farinha de linhaça & 17 & - & 8,5 \\
Linhaça dourada & 3 & - & 1,5 \\
Leite & 10 & 10 & 10 \\
Sal & 0,6 & 0,6 & 0,6 \\
Essência de batata & 0,9 & 0,9 & 0,9 \\
\hline
\end{tabular}

\subsection{ANÁLISE MICROBIOLÓGICA}

As análises microbiológicas foram realizadas por um laboratório de análise de alimentos da cidade de Cascavel-PR, de acordo com os procedimentos descritos na Instrução normativa RDC No 12, de 02 de janeiro de 2001 para Bacillus cereus, Coliformes Termotolerantes a $45^{\circ} \mathrm{C}$, Staphylococcus aureus e Salmonella spp. Os resultados obtidos foram comparados com os parâmetros de normalidade estabelecidos pela legislação vigente.

\subsection{ANÁLISE FÍSICO-QUÍMICA}

As análises físico-químicas foram processadas por um laboratório de análise de alimentos da cidade de Cascavel-PR, onde foram realizadas as análises de gorduras totais, proteína bruta, resíduo mineral fixo (cinzas), umidade e fibra bruta de 
acordo com a metodologia da Instrução Normativa no 93, de 31 de outubro de 2000. As análises foram elaboradas sobre os produtos desenvolvidos e sobre a batata comercial.

\subsection{ANÁLISE SENSORIAL}

Antes do participante iniciar a análise sensorial, os mesmos foram convidados a responder um questionário verbalmente, para avaliar se apresentavam alergia a qualquer componente da formulação dos produtos funcionais similares à batata smile, e em caso positivo o indivíduo era orientado em não participar da análise.

As amostras foram oferecidas em porções de $20 \mathrm{~g}$ cada, em copos codificados com algarismos de três dígitos, com um copo de água para que os participantes realizarem a limpeza do palato. As mesmas foram servidas aleatorizadas, de acordo com o que foi descrito pelos autores Macfie e Bratchell (1989).

Foram avaliados os atributos aceitação global, aparência, aroma, sabor e a textura das amostras, utilizando uma escala hedônica estruturada de nove pontos, cujos extremos de notas variavam entre gostei muitíssimo (9) a desgostei muitíssimo (1). Também foi realizada a avaliação da intenção de compra, porém, para este atributo utilizou-se uma escala estruturada de cinco pontos, cujas extremidades variavam de certamente compraria (5) a certamente não compraria (1).

O índice de aceitabilidade foi calculado a partir das médias e nota máxima dos cinco atributos obtidos, a partir da escala hedônica de 9 pontos.

$\mathrm{IA}=\frac{\mathrm{C} \times 100}{\mathrm{~B}} \rightarrow$ sendo; $\mathrm{C}=$ nota média obtida para o produto

\subsection{ANÁLISE ESTATÍSTICA}

Foi realizada análise de variância ANOVA e as médias foram testadas por Tukey, utilizando-se o programa Microsoft Excel.

\section{RESULTADOS E DISCUSSÕES}

\subsection{PESQUISA DE CONSUMO}

Participaram da pesquisa de consumo 257 entrevistados, de ambos os gêneros, $44 \%$ do gênero masculino e $56 \%$ do gênero feminino, com idade média de $24 \pm 6,1$ anos, consumidores de batatas processadas.

\subsubsection{Consumo de batatas e seus produtos derivados}

Na figura 2, estão apresentadas as informações sobre percentual de consumo pela população dos produtos produzidos a partir da batata processada. Os resultados obtidos revelam que a batata frita $(46,7 \%)$ é o produto mais consumido, seguido de batata cozida (18,7\%), batata palito congelada (14\%) e purê de batata $(8,6 \%)$.

A batata pré-frita é um dos vegetais congelados que mais se destaca no mundo, devido ao seu importante papel no acompanhamento de lanches e pratos rápidos em Fast foods, e isso contribui para o maior consumo. Em 1997, o consumo per capta de batata pré-frita era de $300 \mathrm{~g} / \mathrm{ano}$, evoluindo para $700 \mathrm{~g}$ em 2007, em 2012, o consumo alcançou a 314 mil toneladas, aproximadamente $1,6 \mathrm{Kg}$ por pessoa (RAMOS et al,2013). 
Figura 2. Consumo de batatas e seus produtos derivados

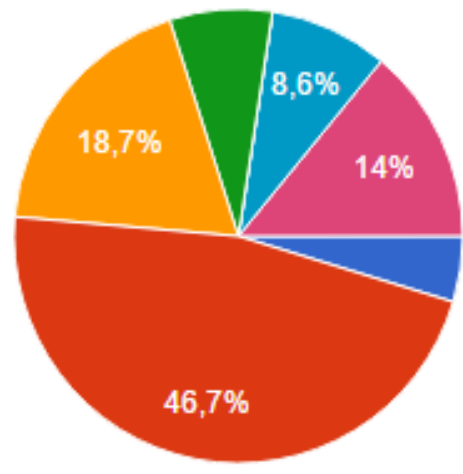

Nenhum

Batata frita

Batata cozida

Batata assada

Batata smile

pure de batata

batata palito congelada

Brognoli (2010) realizou um estudo sobre o consumo de congelados com 180 entrevistados de ambos os gêneros, frequentadores de um supermercado de Criciúma- SC, de acordo com os resultados obtidos, os produtos cárneos $(39,4 \%)$, seguido de pizza $(33,3 \%)$, lasanha $(9,4 \%)$ e batata palito congelada $(9,4 \%)$ foram os produtos congelados mais consumidos no local devido aos principais motivos de praticidade $(51,1 \%)$ e sabor $(30 \%)$.

Segundo Food Trends Brasil, (2010), os produtos que mais despertam desejo quando são lançados no mercado são iogurtes (32\%), bolachas e biscoitos $(28 \%)$, sucos artificiais $(27 \%)$, chocolate e bombons $(25 \%)$, queijos $(24 \%)$, alimentos congelados ou semi prontos (21\%) e arroz (19\%).

De acordo com a figura 3, a maioria dos entrevistados relataram consumir 2 vezes ou mais por semana batatas processadas e seus derivados $(25,3 \%)$, seguido de 1 vez por semana (24,1\%), a cada 15 dias $(22,6 \%)$ e 1 vez por mês $(17,5 \%)$.

Figura 3. Frequência de consumo de batatas processadas e seus derivados

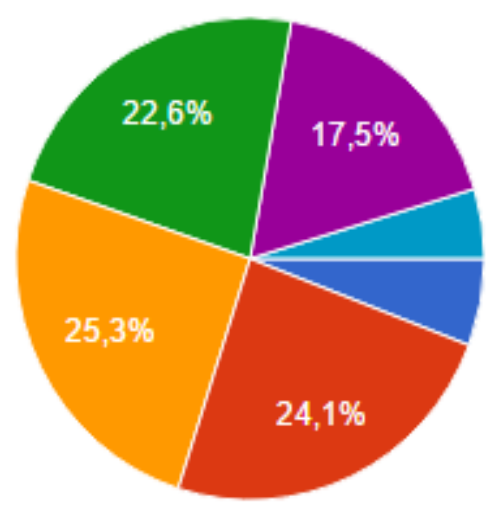

Diário

1 vez por semana

2 vezes ou mais por semana

15 dias

1 vez por mês

não consumo

Silva et al (2016), realizou uma pesquisa com 215 participantes via Google formulário para a verificação do conhecimento em relação à batata in natura e sobre - hábito alimentar dos seus entrevistados. Foi relatado o consumo de aproximadamente $2 \mathrm{~kg}$ de batata congelada por mês.

Cavalcante e Gubert (2015) realizaram uma pesquisa sobre frequência de consumo de alimentos saudáveis e não saudáveis com 360 crianças, entre 1 a 5 anos 
de uma população de baixa renda, foi possível observar que $29,5 \%$ das crianças consumiam de 1 à 3 vezes na semana batata frita ou de pacote.

$\mathrm{Na}$ figura a seguir é possível observar que a maior parte dos consumidores de batatas processadas alega consumir por causa do sabor $(41,2 \%)$, seguido de praticidade $(19,5 \%)$, variar o cardápio $(16,3 \%)$, hábito alimentar $(12,5 \%)$ e pela qualidade $(8,2 \%)$.

Figura 4. Motivo de consumo de batatas processadas

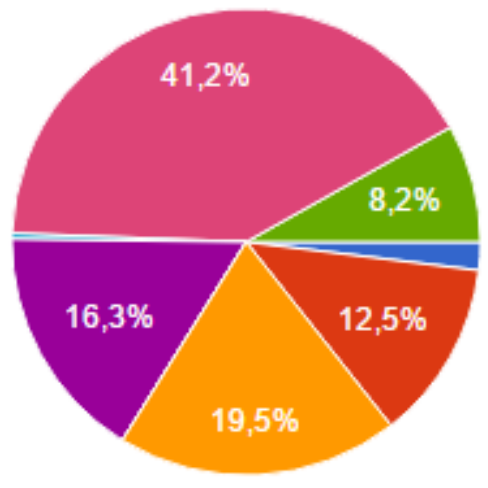

saudável

hábito familiar

praticidade

qualidade

variar o cardápio

preço

sabor

Não consumo

Food Trends Brasil, (2010) ressalta que conveniência e praticidade são as principais tendências em relação ao consumo de alimentos, seguido de confiabilidade e qualidade, sensorialidade e prazer, saudabilidade e bem-estar e sustentabilidade e ética.

Farias (2015), segundo a sua pesquisa realizada com 15 alunos entre 19 a 36 anos, também relata que o principal motivo do consumo de alimentos industrializados e devido a sua praticidade (6,7\%), sendo favorável, pois a falta de tempo $(66,7 \%)$ para o preparo de refeições necessita de algo prático.

Além das perguntas respondidas acima, $69,3 \%$ dos entrevistados relataram achar a batata um alimento saudável. Em relação à batata smile, $74,3 \%$ não consideram a batata smile como um alimento saudável e 13,6\% acham a batata smile um alimento saudável e 12,1\% preferiram não opinar.

Segundo Mori et al (2015), a batata é considerada um tubérculo mais importante para o Brasil, constituindo-se de uma produção anual em torno de 3,5 milhões de toneladas em uma área de aproximadamente 130 mil hectares, com a contribuição de aproximadamente 5 mil produtores em variáveis regiões. Ou seja, a maior parte da produção de batata no Brasil é distribuída de forma in natura, e 10\% é atribuído para a indústria, na produção de batatas pré-fritas congeladas, chips e batata palha.

Sendo um alimento versátil, na culinária pode ser preparada de diversas maneiras, como cozida, em sopas, assada ou em purês, apresentando consumo frequente pela população brasileira, apresenta em sua composição nutricional fonte de carboidratos, fibras, potássio, vitamina A e C (MINISTÉRIO DA SAÚDE, 2014).

\subsubsection{Conhecimento e consumo de ingredientes funcionais}

Os participantes também foram questionados sobre seu conhecimento em relação aos benefícios das fibras e $67,3 \%$ dos entrevistados alegam conhecer. Também verificou-se que $49,8 \%$ dos entrevistados relataram consumir aveia, porém apenas $31,5 \%$ são consumidores de linhaça. 
A aveia e linhaça são consideradas ingredientes funcionais, por este motivo são utilizados na formulação de alimentos, contribuindo para a saúde como na redução de enfermidades e melhor qualidade de vida (SILVA et al, 2016).

Sobre o consumo de alimentos modificados e/ou enriquecidos, $40,1 \%$ dos entrevistados constataram consumir estes alimentos. $81,7 \%$ dos entrevistados relataram acreditar que os alimentos funcionais podem trazer benefícios à saúde $\mathrm{e}$ $15,2 \%$ disseram que talvez acreditem que os alimentos funcionais podem trazer benefícios à saúde.

Food Trends Brasil (2010), realizou uma pesquisa sobre o conhecimento dos consumidores sobre alimentos funcionais e enriquecidos, entre outros, cujo $8 \%$ dos entrevistados disseram conhecer bem sobre alimentos funcionais, enquanto $22 \%$ relatam conhecer pouco, $30 \%$ só ouviram falar e $40 \%$ não conhecem ou nunca ouviram falar. Para o termo alimentos enriquecidos, $17 \%$ dizem conhecer bem sobre alimentos enriquecidos enquanto $35 \%$ relatam conhecer pouco, $31 \%$ só ouviram falar e $17 \%$ não conhecem ou nunca ouviram falar.

Neste contexto, o desenvolvimento de alimentos com ingredientes contendo propriedades funcionais tem um nicho de mercado, especialmente no que diz respeito às batatas funcionais, porém, para isto é necessário estudo sobre a aceitabilidade deste produto.

\subsection{ANÁLISE MICROBIOLÓGICAS}

Para Fischer (2013) falhas múltiplas podem desencadear surtos alimentares, como uso de matéria-prima e refrigeração inadequada, manipuladores contaminados, processo térmico insuficiente, contaminação cruzada, higienização incorreta, local inadequado e conservação sob temperatura incorreta.

Os resultados da análise microbiológicas dos produtos funcionais similares à batata smile estão dispostos na Tabela 02.

Tabela 2. Análises microbiológicas realizadas em amostras de produtos funcionais similares à batata smile com adição de diferentes concentrações de linhaça e aveia na formulação, sendo BFL (somente farinha de linhaça e linhaça dourada), BFA (somente farinha de aveia e aveia em flocos) e BFLA ( $50 \%$ aveia e $50 \%$ linhaça).

\begin{tabular}{ccccc}
\hline Formulação & $\begin{array}{c}\text { Bacillus cereus } \\
1,0 \times 10^{3} \mathrm{UFC} / \mathrm{g}\end{array}$ & $\begin{array}{c}\text { Coliformes Termotolerantes } \\
\text { a } 45^{\circ} \mathrm{C} \\
2,0 \times 10 \mathrm{UFC} / \mathrm{g}\end{array}$ & $\begin{array}{c}\text { Staphylococcus } \\
\text { aureus } \\
1,0 \times 10^{3} \mathrm{UFC} / \mathrm{g}\end{array}$ & $\begin{array}{c}\text { Salmonella spp. } \\
\text { Ausência em } 25 \mathrm{~g}\end{array}$ \\
\hline BFL & $<1,0 \times 10^{2}$ & $<1,0 \times 10^{1}$ & $<1,0 \times 10^{2}$ & Ausência \\
BFA & $<1,0 \times 10^{2}$ & $<1,0 \times 10^{1}$ & $<1,0 \times 10^{2}$ & Ausência \\
BFLA & $<1,0 \times 10^{2}$ & $<1,0 \times 10^{1}$ & $<1,0 \times 10^{2}$ & Ausência \\
\hline
\end{tabular}

Os dados microbiológicos mostraram que os produtos desenvolvidos apresentaram resultados compatíveis com os exigidos pela legislação (BRASIL, 2001).

\subsection{ANALISE FÍSICO-QUÍMICA}

$\mathrm{Na}$ tabela 3 estão apresentados os resultados da composição centesimal dos produtos funcionais similares à batata smile. 
Tabela 3. Análise físico-química em amostras de produtos funcionais similares à batata smile com adição de diferentes concentrações de linhaça e aveia na formulação, sendo BFL (somente farinha de linhaça e linhaça dourada), BFA (somente farinha de aveia e aveia em flocos) e BFLA ( $50 \%$ aveia e $50 \%$ linhaça).

\begin{tabular}{ccccc}
\hline & BFL & BFA & BFLA & BC $^{\star}$ \\
\hline Carboidrato(g) & 27,28 & 31,5 & 28,7 & 31,7 \\
Proteína(g) & 8,6 & 7,1 & 8,2 & 2 \\
Lipídio(g) & 6,5 & 2,4 & 6,6 & 6 \\
Fibras(g) & 4,4 & 1,9 & 4,6 & 0,7 \\
Umidade & 54,4 & 56,26 & 53,32 & - \\
Cinzas & 3,15 & 2,70 & 3,12 & -
\end{tabular}

* Informação obtida através do rótulo nutricional do produto.

Os resultados das amostras de produtos funcionais similares à batata smile com adição de linhaça dourada e aveia, comparada ao produto convencional, mostraram-se com teor de carboidrato da BFL reduzido em 8,6 vezes, para BFA reduziu em 9,9 vezes e BFLA reduziu em 9 vezes. Segundo Azevedo (2013) o aumento da obesidade no Brasil deve-se, principalmente, ao consumo excessivo de alimentos hipercalóricos e diminuição do consumo de alimentos ricos em fibras, sendo um dos maiores fatores de risco para doenças crônicas não transmissíveis. 0 consumo excessivo de sódio pela alimentação é um dos principais fatores de risco para o desenvolvimento de hipertensão. Dados da vigilância de fatores de risco e proteção pra doenças crônicas por inquérito telefônico (VIGITEL), do Ministério da Saúde, apontam que $24,8 \%$ da população adulta apresentam diagnóstico médico de hipertensão arterial.

Os produtos desenvolvidos comparados ao comercial apresentaram também aumento do teor de proteína, para BFL em 4,3 vezes, para BFA em 3,5 vezes e para BFLA em 4,1 vezes, o que é explicado pelo grande teor deste macronutriente presente na linhaça e na aveia (COLUSSI, 2013). Sendo a proteína um macronutriente necessário para a formação, desenvolvimento e crescimento de tecidos corporais, entre outros benefícios.

As formulações a base de linhaça dourada apresentaram valores maiores de lipídio e fibras em relação à formulação com adição de aveia e ao produto comercial. A formulação BFL teve aumento lipídico de 1,0 vezes comparado ao produto comercial (BC), a formulação BFA teve redução lipídica de 4,0 vezes comparada a BC e BFLA teve aumento lipídico de 1,1 vez comparado a $B C$. Em relação às fibras, a formulação BFL teve aumento de 6,3 vezes, a BFA teve aumento de 2,7 vezes e BFLA teve um aumento de 6,5 vezes em relação à $\mathrm{BC}$.

O percentual de lipídio aumentou de acordo com o aumento da quantidade de linhaça, já que a mesma é rica em gordura poli-insaturada que é benéfica ao organismo (MACÊDO, 2014). Segundo Novello e Pollonio (2012), a ingestão, principalmente de ácido graxo a-linolênico, pode reduzir a incidência de doenças cardiovasculares. Os mesmos possuem funções importantes para a manutenção da saúde humana, são necessários para a manutenção da fluidez, flexibilidade e permeabilidade seletiva da membrana celular, atuam nas funções cerebrais e 
transmissão de impulsos nervosos, na síntese de hemoglobina, auxiliam no transporte de vitaminas lipossolúveis, entre outras funções (RIBEIRO, 2012).

Outros autores também produziram alimentos enriquecidos com aveia e linhaça, Colussi et. al. (2013), desenvolveu barras de cereais a base de aveia e linhaça dourada, obtendo significativo aumento de fibras e proteínas, principalmente nas barras de cereais produzidas com maior adição de linhaça. Gutkoski et al. (2007), desenvolveu barras de cereais com diferentes concentrações de farelo de aveia como substituto parcial da farinha, obtendo um produto com maior quantidade de proteína e fibras. Macêdo et al (2014), produziu biscoitos salgados isentos de glúten com adição de linhaça em diferentes concentrações, para os valores de proteína não foi observado diferença significativa entres os produtos e para valores de fibras, foi considerado um alimento com boa fonte de fibra alimentar.

\subsection{ANÁLISE SENSORIAL}

Participaram da análise sensorial 134 provadores, de ambos os gêneros, $34 \%$ do gênero masculino e $66 \%$ do gênero feminino, com idade média de $21 \pm 5,1$ anos, consumidores de batatas processadas. Os resultados obtidos a partir da análise estão representados na Tabela 4 a seguir.

Tabela 4. Médias de aceitação em amostras de produtos funcionais similares à batata smile com adição de diferentes concentrações de linhaça e aveia na formulação, sendo BFL (somente farinha de linhaça e linhaça dourada), BFA (somente farinha de aveia e aveia em flocos) e BFLA (50\% aveia e $50 \%$ linhaça).

\begin{tabular}{lcccccc}
\hline Atributos & BFL & BFA & BFAL & BC & DMS & Valor de P \\
\hline Ac. Global & $5,4 \pm 2,1^{\mathrm{C}}$ & $6,2 \pm 1,8^{\mathrm{B}}$ & $5,7 \pm 2,0^{\mathrm{C}}$ & $8,2 \pm 0,8^{\mathrm{A}}$ & 0,408 & $<0,0001$ \\
Aparência & $5,9 \pm 2,0^{\mathrm{C}}$ & $6,4 \pm 1,9^{\mathrm{BC}}$ & $6,4 \pm 1,8^{\mathrm{B}}$ & $8,2 \pm 1,1^{\mathrm{A}}$ & 0,418 & $<0,0001$ \\
Aroma & $5,4 \pm 2,1^{\mathrm{C}}$ & $6,0 \pm 1,9^{\mathrm{B}}$ & $5,7 \pm 2,0^{\mathrm{BC}}$ & $7,9 \pm 1,2^{\mathrm{A}}$ & 0,438 & $<0,0001$ \\
Sabor & $4,5 \pm 2,3^{\mathrm{BC}}$ & $5,9 \pm 2,2^{\mathrm{B}}$ & $5 \pm 2,2^{\mathrm{C}}$ & $8,2 \pm 0,9^{\mathrm{A}}$ & 0,497 & $<0,0001$ \\
Textura & $5,7 \pm 2,3^{\mathrm{C}}$ & $6,6 \pm 1,9^{\mathrm{B}}$ & $6,1 \pm 1,9^{\mathrm{BC}}$ & $8,1 \pm 1,0^{\mathrm{A}}$ & 0,450 & $<0,0001$ \\
\hline
\end{tabular}

Nas linhas, letras maiúsculas iguais indicam que estatisticamente as amostras são

iguais, ao passo que letras maiúsculas diferentes indicam que as amostras diferem estatisticamente.

Segundo a análise de variância (ANOVA), todos os atributos como a aceitação global (AG), aparência, aroma, sabor e textura apresentaram diferenças significativas $(p<0,05)$. A partir do teste de Tukey foi constatado que as amostras diferem entre elas, além de ter observado a preferência pela batata comercial (BC) em todos os atributos avaliados. Das batatas funcionais avaliadas, a que apresentou adição de linhaça (BFL), obteve menor preferência entre as amostras.

A amostra (BFL) obteve menor aceitação pelo seu provável sabor amargo, em virtude do óleo presente, além da sua coloração alaranjada (WIGNEZ; PIVARO, 2012). Apesar destas características, a linhaça é uma das fontes de óleo vegetal, fonte de ômega 3, auxiliando na redução de infarto do miocárdio, trombose, gangrena, redução do colesterol LDL, total e ação antioxidante entre outros (WIGNEZ; PIVARO, 2012).

Torres e Casarotti (2016) elaboraram biscoitos com farinha de linhaça e biscoitos sem linhaça para a verificação da aceitabilidade, foi concluído que o produto obteve uma boa aceitação, além de oferecer maior maciez e aumento no valor nutritivo do produto.

Ritter (2014) desenvolveu pão de mel com adição de farinha de linhaça e outros tipos de produtos que apresentam fibras em sua composição, como fibra de 
soja, farelo de trigo e farinha de centeio. Utilizando escala hedônica de expressão facial de cinco pontos para crianças de 6 a 10 anos, 69\% disseram ter gostado do produto enquanto $28 \%$ gostaram, $2 \%$ indiferente e $1 \%$ não gostaram.

Além de adicionar a farinha de linhaça em preparações como biscoito e pão de mel, é possível introduzir na produção de bolo de casca de laranja. A introdução da linhaça apresentou apenas influência sobre a cor do bolo, o produto desenvolvido apresentou uma boa aceitação sobre os provadores (SILVA; TOBAL,2014).

Colussi et al (2013), produziram 5 amostras de barras de cereais elaboradas a base de linhaça dourada e aveia (100\% aveia, 100\% linhaça dourada, 50\% aveia e linhaça dourada, $25 \%$ de aveia e $75 \%$ de linhaça dourada e $75 \%$ de aveia e $25 \%$ de linhaça dourada), a amostra com $50 \%$ de aveia e linhaça obteve maior aceitabilidade.

As autoras Piovesana e Klajn (2013), produziram 3 amostras de biscoitos enriquecidos com aveia e farinha de bagaço da uva, utilizando $70 \%, 60 \%$ e $50 \%$ de farinha de trigo, $15 \%, 20 \%$ e $25 \%$ de farinha integral de aveia e farinha de bagaço de uva, o resultado das amostras não diferiram significativamente entre elas. $\mathrm{O}$ biscoito, com adição de $70 \%$ de farinha de trigo e $15 \%$ de farinha integral de aveia e farinha de bagaço de uva, obteve maior aceitação sobre todos os atributos como sabor, crocância e impressão global. Deste modo, é possível observar o aumento da introdução da aveia e da linhaça em farinhas mistas em conjunto com a farinha de trigo, devido a sua composição nutricional, considerada de alta qualidade, constituindo-se de $13,23 \%$ de proteína e $6,38 \%$ de fibra alimentar, sendo benéfica à saúde (CARNEIRO, 2015).

De acordo com os resultados do índice de aceitabilidade, verificou-se que a batata comercial (BC) apresentou $90 \%$ de aceitabilidade seguida de batata funcional com adição de aveia (BFA) com $69 \%$, batata funcional com adição de aveia e linhaça (BFAL) com $64 \%$ e a batata funcional com adição de linhaça (BFL) com $60 \%$ apresentou menor aceitabilidade.

Bastos et al. (2014) ressaltam que para o desenvolvimento de um novo produto, avaliar a sua aceitabilidade é um dos fatores fundamentais, por este motivo o mesmo relata que o produto deve atingir no mínimo $70 \%$ de índice de aceitabilidade para que seja considerado aceito segundo as suas propriedades sensoriais. Com isto, é possível observar que a amostra BFA ficou com índice de aceitabilidade muito próximo do recomendado. Porém, as outras formulações tiveram índice de aceitabilidade inferior e, portanto, necessitam de significativa melhora na formulação do produto. Na Tabela 5 segue o índice de aceitabilidade das amostras testadas.

Tabela 5. Índice de aceitabilidade em amostras de produtos funcionais similares à batata smile com adição de diferentes concentrações de linhaça e aveia na formulação, sendo BFL (somente farinha de linhaça e linhaça dourada), BFA (somente farinha de aveia e aveia em flocos) e BFLA ( $50 \%$ aveia e $50 \%$ linhaça).

\begin{tabular}{lcccc}
\hline Atributos & BFL & BFA & BFAL & BC \\
\hline Ac. Global & 60 & 69 & 63 & 91 \\
Aparência & 65 & 71 & 71 & 91 \\
Aroma & 60 & 66 & 63 & 88 \\
Sabor & 50 & 65 & 55 & 91 \\
Textura & 63 & 73 & 68 & 90 \\
Média IA\% & $\mathbf{6 0 \%}$ & $\mathbf{6 9 \%}$ & $\mathbf{6 4 \%}$ & $\mathbf{9 0 \%}$ \\
\hline
\end{tabular}




\subsection{INTENÇÃO DE COMPRA}

A figura abaixo apresenta o resultado da intenção de compra, pode se observar que 81 participantes relataram que certamente comprariam (5) a Batata comercial (BC), enquanto a Batata funcional com adição de linhaça (BFL), apresentou apenas 9 participantes que relataram que certamente comprariam, sendo a preparação com maior rejeição.

Figura 5. Intenção de compra em amostras de produtos funcionais similares à batata smile com adição de diferentes concentrações de linhaça e aveia na formulação, sendo BFL (somente farinha de linhaça e linhaça dourada), BFA (somente farinha de aveia e aveia em flocos) e BFLA (50\% aveia e $50 \%$ linhaça)

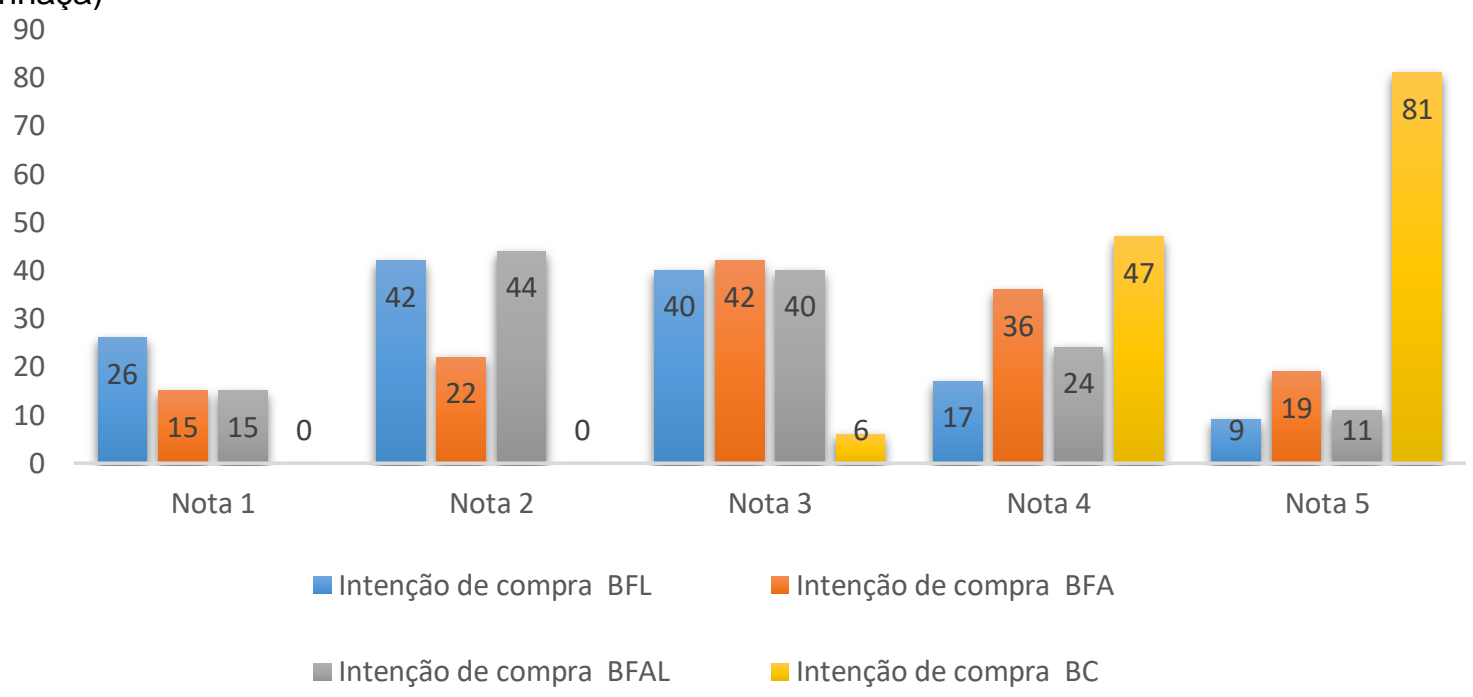

Segundo Lugoboni (2013), o desenvolvimento de produtos define como colocar em prática a ideia para que seja concretizada em estrutura física ou um serviço a ser prestado. Considerando assim, o desenvolvimento de novos produtos como um fator de extrema importância para o crescimento das empresas devido à progressiva internacionalização dos mercados, relacionado ao aumento da variedade e diversidade de ofertas, além da redução do ciclo de vida dos produtos (PLENTZ et al, 2014).

Devido à busca por uma alimentação saudável para a melhoria da qualidade de vida, aumenta a possibilidade de elaboração de produtos inovadores tais como; produtos sem adição de aditivos alimentares, ou seja, "naturais", alimentos modificados e enriquecidos com vitaminas e minerais, produtos diminuidores de apetites, entre outros (LAUSCHNER et al, 2016).

\section{CONSIDERAÇÕES FINAIS}

Foi verificado o maior consumo sobre a batata frita entre os processados de batata, sendo o sabor e praticidade os motivos de maior relevância para o consumo dos mesmos. Os entrevistados apresentaram bom conhecimento sobre os benefícios das fibras, relatando o maior consumo de aveia e menor consumo de linhaça e alimentos enriquecidos e/ou modificados. A maior parte dos entrevistados relatam acreditar que os alimentos funcionais podem trazer benefícios à saúde.

Foi possível elaborar um produto a base de batata semelhante ao convencional, porém, com redução dos teores de sódio e carboidrato, e com aumento dos teores de proteína, fibras e lipídios de boa qualidade nutricional. Em relação à análise sensorial, foi possível observar a grande preferência sobre a batata comercial, 
devendo realizar modificações na formulação das demais amostras similares a batata smile, para a maior satisfação ao público.

Por fim, conclui-se que é de extrema relevância a produção de um alimento inovador como a batata funcional pré-assada com adição de grãos, devido a sua praticidade de consumo e maior valor nutricional empregado a mesma.

\section{REFERÊNCIAS}

AGÊNCIA NACIONAL DE VIGILÂNCIA. Regulamento técnico que estabelece as diretrizes básicas para análise e comprovação de propriedades funcionais e ou saúde alegadas em rotulagens de alimentos. Resolução no 18, 1999.

AGROCLUSTER, R. Tendências do mercado alimentar do Brasil: Aspecto do consumo, produto, distribuição e comunicação. Janeiro.2015.

AZEVEDO, E. C. de C., Consumo alimentar de risco e proteção para doenças crônicas não transmissíveis e sua associação com a gordura corporal: um estudo em funcionários da área de saúde de uma universidade pública de Recife - PE. 2013. Ciênc. saúde coletiva. 2014, vol.19, n.5, pp.1613-1622. ISSN 14138123. http://dx.doi.org/10.1590/1413-81232014195.06562013.

BERNAUD, F.S.R.; RODRIGUES, T.C. Fibra alimentar- Ingestão adequada e efeitos sobre a saúde do metabolismo. Rev. Arq Bras Endocrinol Metab. Porto alegre, RS, n.57/6, p. 397-405, 14 . abr. 2013. http://dx.doi.org/10.1590/S000427302013000600001

BROGNOLI, M.L. Consumo de alimentos congelados por indivíduos frequentadores de supermercados. 2010. Dissertação (Trabalho de conclusão de curso) - Universidade do extremo sul catarinense - UNESC, Criciúma- SC.

CARNEIRO, G.S.; PIRES, C.R.F.; PEREIRA, A. S.; CUNHA, N.T.; SILVA, C. A. Caracterização físico-química de bolos com substituição parcial da farinha de trigo por aveia, quinoa e linhaça. Enciclopédia biosfera, centro cientifico conhecer. Goiânia, vol 11, n.21, p.3353, 01 jun.2015.

CAVALCANTE, I.S.; GUBERT, M.B. Frequência de consumo de alimentos marcadores de alimentação saudável e não saudável entre crianças de 1 a 5 anos em uma população de baixa renda no Distrito Federal. 2015. Dissertação (Trabalho de Conclusão de Curso) Brasília.

COLUSSI, R.; BALDIN, F.; NOELLO, C.; HARTMANN, V.; GUTKOSKI, L.C. Aceitabilidade e estabilidade físico-química de barras de cereais elaboradas à base de aveia e linhaça dourada. Rev. Braz. J. Food Techonol, Campinas, v.16, n.4, p.292-300, out/dez. 2013. http://dx.doi.org/10.1590/S1981-67232013005000035.

COSTA, N.M.B.; ROSA, C.O.B. Alimentos funcionais: Componentes bioativos e efeitos fisiológicos. 2 ed. Rio de Janeiro. Rubio,2016.

DUTCOSKY, S.D. Analise sensorial de alimentos. 4ed. Curitiba. Champagnat, 2013. 
EMBRAPA. Aspectos econômicos e conjunturais da cultura da aveia. Rio grande do sul. 2012. Disponível em < https://www.embrapa.br/busca-de-publicacoes//publicacao/969145/aspectos-economicos-e-conjunturais-da-cultura-da-aveia>

Acesso em: 28.04.2017.

FARIAS, L.J.S. Consequências do consumo excessivo de alimentos industrializados, ricos em cloreto de sódio no IFMA campus Açailândia. Natal, RN. 2015.Disponivel em < http://www.abq.org.br/sinequi/2015/trabalhos/100/651220178.html> Acesso em: 27. Abr. 2017.

FOOD TRENDS BRASIL 2020. São Paulo. 2010. Disponível em $<$ http://www. alimentosprocessados.com.br/arquivos/Consumo-tendencias-einovacoes/Brasil-Food-Trends-2020.pdf > Acesso em: 27. Abr. 2017.

LAUSCHNER, D.S.; LINN, A.L.; RUSCHIEL, T.C.; ALBERTI, R.A.; JÚNIOR, J.F.; ILHA, J.; PILETTI, R. Desenvolvimento de novos produtos alimentícios: Hambúrguer recheado. In: $3^{\text {o }}$ Simpósio de Agronomia e Tecnologia de Alimentos (AGROTEC), 2016, Itapiranga- SC.

LUGOBONI et al. Lançamento de novos produtos no segmento alimentício B2B: uma comparação as ações realizadas pelo mundo. In: $3^{\circ}$ simpósio de agronomia e tecnologia de alimentos. Salvador- BA, 2013.

LUZIETTO, E.M.; TURECK, C.; LOCATELI, G.; CORREA, V.G.; KOEHNLEIN, E.A. Alimentos funcionais em alimentação coletiva: reflexões acerca da promoção da saúde fora do domicilio. Rev. Nutrite. Maringá- PR, Aug:40(2), p. 188-199. 2015. http://dx.doi.org/10.4322/2316-7874.49714

MACÊDO, P. M. S., et al., Avaliação físico química e sensorial de biscoito salgado isento de glúten contendo farinha de linhaça. Rev. Tecnológica, Maringá. 2014.

MACFIE, H.J.; BRATCHELL, NICHOLAS. Designs to balance the effect of order of presentation and first-order carry-over effects in hall tests. Journal of Sensory Studies n.4. p.129-148, 7. ago.1989. https://doi.org/10.1111/j.1745459X.1989.tb00463.x

MARÇAL, M.L.; BERNARDI.M.D. Produção de batata funcional pré-assada: Análise microbiológica, físico-quimica e nutricional. 2017 Dissertação (Trabalho de Conclusão de Curso) - Centro Universitário Assis Gurgacz, Cascavel-PR.

MINISTÉRIO DA SAÚDE. Guia alimentar para a população Brasileira. 2e․ 1. reimpr.Brasília. 2014.

MORI, C.; FONTANELI, R.S.; SANTOS, H. P. Sistema de produção da batata. Ministério da agricultura, pecuária e abastecimento. Passo fundo, RS.2 ed. ISSN 1518-6512, agosto.2015.

NETO, A.M.Á. Ministério do desenvolvimento, indústria e comércio exterior secretaria de comércio exterior. Circular № 22, 11. abr, 2016. 
NEVES, L.C.M.N. Oferta de alimentos processados na universidade de Brasília. 2016. Dissertação (Trabalho de Conclusão de Curso) -Universidade de Brasília, faculdade de ciências da saúde departamento de nutrição, Brasília.

NOBRE.M.F., Produção de pão a partir de farinhas estremes de aveia. Universidade de Lisboa, 2013.

NOVELLO, D.; POLLONIO, M. A. R. Caracterização físico química e microbiológica da linhaça dourada e marrom. Rev Inst Adolfo Lutz. 2012

PILAR.B.C. Avaliação dos efeitos da semente de Linum usitatissimum L. em pacientes com síndrome metabólica. 2014. Dissertação (Mestrado em bioquímica) - Universidade Federal do Pampa, Uruguaiana, RS.

PIOVESSANA, A.; BUENO, M.M.; KLAJN, V.M. Elaboração e aceitabilidade de biscoitos enriquecidos com aveia e farinha de bagaço de uva. Rev. Braz. J. Food technol. Campinas, vol. 16, n.1, p.68-72, 2013. http://dx.doi.org/10.1590/S198167232013005000007

PLENTZ, N.D.; ANDRADE, P.D.; ZEN.A.C.; MOREIRA, M.; BERNARDES, S. Análise de práticas no desenvolvimento de novos produtos: estudos de casos múltiplos em empresas de bens de consumo. In: XXXIV Encontro nacional de engenharia de produção Engenharia de Produção, 2014, Curitiba-PR.

RAMOS, R.M.; SILVA, A.R.; DUMBRA, J.G.R. O aquecido mercado dos vegetais congelados. Rev. Hortifruti Brasil. n. 122, ISSN 1981- 1837, abr. 2013.

RIBEIRO, L. F. Uso de espectroscopia no infravermelho e análise multivariada para previsão de ácidos graxos em linhaça dourada e marrom. Curitiba, 2012.

RITTER.A.M. Pão de mel integral de fibras. 2014. Dissertação (Trabalho de Conclusão de Curso) - Centro Universitário Univates, Lajeado.

SCHULKA. A. C.; COLLA, E.X. Alimentos funcionais e suas perspectivas no mercado Brasileiro. Rev. Food Ingredients Brasil, ㄲo30. 2014.

SILVA, A.C.C.; SILVA, N.A.; PEREIRA, M.C.S.; VASSIMON, H. S. Alimentos contendo ingredientes funcionais em sua formulação: revisão de artigos publicados em revistas brasileiras. Rev. conexão e ciência. São Paulo, vol.11. no2.p. 133-144, 07. dez. 2016. https://doi.org/10.24862/cco.v11i2.429

SILVA, J.S.; TOBAL, T.M. Utilização da casca de laranja e farinha de linhaça na produção de bolos: influencia na aceitação e no valor nutritivo. In: ENEPEX: Encontro de Ensino, Pesquisa e Extensão. 2014.

SILVA, P.N.L.; JUNIOR, R.F.; SANTOS, E.F. Conhecimento do consumidor e forma de apresentação da batata no mercado no estado de São Paulo. Rev. iPecege. São Paulo, 2(2), p 46-55, 13. maio. 2016. DOI: 10.22167/r.ipecege.2016.2.46 
SIMONATTO, M. Produção de silagem pré-secada com diferentes gramíneas de clima temperado. 2013. Dissertação (Trabalho de Conclusão de Curso de Zootecnia) - Universidade Tecnologia Federal do Paraná, Dois Vizinhos - PR.

STRANCK, L.T. Crescimento, desenvolvimento e produtividade de linhaça marrom e dourada nas condições e da foclimáticas de curitibanos-SC. 2015. Dissertação (Trabalho de Conclusão de Curso de Agronomia) - Universidade Federal de Santa Catarina, Curitiba-PR.

TORRES, A.; CASAROTTI, S.N. Analise sensorial de biscoitos elaborados com farinha de linhaça. UNILAGO. 2016.

WIGNEZ, H.; PIVARO, T.M. O óleo de linhaça na alimentação de bovinos. Rev. Pesquisa \& tecnologia. 2012. 